Service social

\title{
La garde des enfants suite au divorce : contexte historique, courants actuels et perspectives empiriques
}

\section{Madeleine Beaudry}

Volume 37, numéro 3, 1988

Index analytique 1978-1988

URI : https://id.erudit.org/iderudit/706413ar

DOI : https://doi.org/10.7202/706413ar

Aller au sommaire du numéro

Éditeur(s)

École de service social de l'Université Laval

ISSN

1708-1734 (numérique)

Découvrir la revue

Citer cet article

Beaudry, M. (1988). La garde des enfants suite au divorce : contexte historique, courants actuels et perspectives empiriques. Service social, 37(3), 435-454. https://doi.org/10.7202/706413ar d'utilisation que vous pouvez consulter en ligne. 


\section{COMMENTAIRES \\ ET DOCUMENTS}

BEAUDRY, Madeleine, étudiante au doctorat en service social, Université Laval.

\section{La garde des enfants suite au divorce : contexte historique, courants actuels et perspectives empiriques}

\section{Madeleine Beaudry}

Au cours des dernières décennies, le nombre d'enfants touchés par le divorce de leurs parents n'a cessé de croître. Selon Statistique Canada (1986), 83000 enfants canadiens et 40000 québécois sont impliqués annuellement dans une réorganisation familiale. Or, on constate que les femmes obtiennent la garde légale de plus des trois quarts des enfants $(77.2 \%$ par rapport à $15.6 \%$ pour les hommes en 1982). Comment expliquer ce phénomène? Quelles valeurs ou croyances justifient une telle proportion? Se pourrait-il que les orientations privilégiées par notre société ne reposent pas sur une analyse scientifique des conséquences des différentes formes de garde? Quels sont les avantages et les inconvénients reliés à ces types de garde?

Afin d'apporter un éclairage nouveau sur ces questions, nous ferons d'abord un bref historique du concept de garde. Puis, nous verrons comment les transformations de la société contemporaine ont amené les tribunaux à modifier la loi. Enfin, par une revue de la littérature scientifique, nous préciserons les intérêts respectifs des 
enfants, des hommes et des femmes, selon que les parents obtiennent une garde conjointe ou unique.

\section{Contexte historique et valeurs sous-jacentes}

Un bref retour sur l'histoire des différentes formes de garde des enfants au moment de la séparation des parents et l'identification des changements sociaux majeurs dans la société contemporaine nous aideront à mieux comprendre la philosophie et les concepts sousjacents aux lois et aux pratiques actuelles dans ce domaine.

De la priorité du père à celle de la mère. Dans les pays occidentaux, les lois concernant la garde des enfants plongent leurs racines dans la tradition gréco-romaine. On considérait alors que les enfants étaient la propriété du père qui avait même le droit de les vendre ou de les condamner à mort. Le "Common Law " anglais partageait cette philosophie de l'autorité paternelle et, devant un problème de garde des enfants, le résolvait arbitrairement à la faveur du père (Derdeyn, 1978).

Au début du dix-neuvième siècle, l'industrialisation provoque de nombreux changements sociaux, notamment une nouvelle répartition du travail, la modification des rôles parentaux et l'apparition de la famille nucléaire comme fondement de la société (Elkin, 1987). Les pères doivent s'absenter de la maison pour travailler en usine et les mères deviennent les principales responsables des soins et de l'éducation des enfants. Cette responsabilité leur apporte une nouvelle reconnaissance sociale. Les femmes obtiennent le droit de propriété et, en 1839, moment que nous pourrions qualifier d'historique, le Parlement anglais adopte le " Talfourd Act " qui reconnaît à la mère le droit de garde de ses enfants âgés de moins de sept ans.

À cette période, les théories du développement de l'enfant et plus particulièrement la doctrine de "I'âge tendre " (tender years), accordent un rôle prépondérant aux soins maternels prodigués à l'enfant dans son jeune âge. Lentement mais sûrement, le pendule va osciller : la présomption de garde des enfants en faveur du père, établie depuis des siècles, se dissipe au profit de la mère. Pendant plusieurs années, la majorité des gardes lui seront confiées, à moins qu'elle ne soit jugée incapable d'assumer sa tâche.

De la priorité de la mère à la notion de garde conjointe. Tout comme les changements sociaux du dix-neuvième siècle ont entraîné des changements de mentalité par rapport à la garde des enfants, les bouleversements profonds de la société contemporaine continuent à remettre en question les pratiques de garde. Examinons quelques changements qui nous semblent les plus significatifs. 
La courbe quasi exponentielle représentant l'augmentation du nombre de divorces au cours des dernières années est un des signes majeurs de changement. En effet, au Canada comme dans la majorité des pays occidentaux, le taux de divorce augmente de manière fulgurante : $500 \%$ depuis 1968 (Laroche, 1985). Bien qu'il soit périlleux de brosser un tableau exact de la situation des séparations parentales au Canada et au Québec (les données officielles sont incomplètes et les statistiques ne comptent que les jugements définitifs de séparation légale et de divorce), nous avons suffisamment de données pour affirmer l'ampleur du phénomène.

Depuis l'établissement de la loi canadienne sur le divorce en 1968, le nombre des divorces enregistrés au Canada est passé de 30000 en 1970 à près de 69000 en 1983, ce qui représente plus du double de 1970 (Statistique Canada, 1985). Par la suite, en 1983, le nombre de divorces a cependant diminué de près de $2000^{1}$. Toutefois, suite à la nouvelle loi du divorce de 1986 simplifiant les motifs de divorce et réduisant de trois à un an la période de non cohabitation invoquée pour justifier le divorce, une nouvelle augmentation est prévue. De façon générale, les statistiques actuelles de démographie sociale permettent de prévoir qu'une famille sur trois, fondée durant les années 1980, connaîtra une séparation ou un divorce (Gauthier et coll., 1982).

Ce grand nombre de divorces et de séparations touchent de plus en plus d'enfants. Au Canada, plus d'un demi-million d'enfants dépendants ont été affectés par le divorce de leurs parents durant les dernières décennies. Certaines études sur l'augmentation du taux de divorce prévoient que dans la société nord-américaine de 1990, la moitié des enfants connaîtront la séparation de leurs parents (Glick, 1979; Kurdek, 1983).

Cette augmentation de la fréquence du divorce ne peut plus être considérée comme un fait social isolé. Au contraire, c'est un phénomène social complexe, au confluent de multiples changements qui, vus sous l'angle de la théorie des systèmes, s'inter-influencent. Mentionnons par exemple l'urbanisation et l'industrialisation croissantes $^{2}$, l'augmentation du contrôle des naissances et la baisse importante du taux de natalité, l'espérance de vie accrue, la remise en question du rôle de la famille et du couple par rapport à la satisfaction des besoins individuels, la scolarisation accrue, la prépondérance des droits individuels, l'accent sur les valeurs hédonistes, I'apparition de lois facilitant l'accès au divorce, la diminution du rejet social face aux divorcés (Irving et Benjamin, 1986).

Parmi ces profondes modifications de la société contemporaine, il faut reconnaître l'importance de l'entrée des femmes sur le marché du travail et leur prise de conscience collective de la place qui leur est 
réservée. Davantage conscientes des inégalités sociales dont elles sont victimes et aspirant à plus d'égalité entre les sexes, plus de femmes remettent en question les rôles traditionnels, poursuivent leurs études et souhaitent une carrière ou un travail rémunérateur en vue d'une plus grande autonomie. Par exemple, en 1985 au Canada, $62.6 \%$ des femmes de 25 à 34 ans occupent un emploi (Comité de la Consultation sur la politique familiale, 1987, p. 21), $56 \%$ des mères d'enfants d'âge préscolaire font partie de la population active et $22 \%$ d'entre elles travaillent à temps partiel (Statistique Canada, 1985). Devant leur double tâche, est-il surprenant de constater que plus de femmes aspirent à une plus grande équité dans le partage des responsabilités parentales?

Un autre facteur important dans l'évolution des mentalités par rapport à la garde des enfants est relié à la valorisation du rôle du père. Les progrès de la psychologie contemporaine mettent en évidence l'importance du père dans le développement de l'enfant (Keshet, 1978) et remettent en question le fait que seule la mère peut s'occuper adéquatement de son enfant en raison de son instinct maternel (doctrine de l'âge tendre). La théorie du rôle, selon laquelle le comportement est prescrit par la situation sociale, puis dirigé par les motivations individuelles et les valeurs de la société (Strean, 1983), laisse plutôt entrevoir que les rôles joués jusqu'alors par les hommes et les femmes auprès des enfants répondaient à des impératifs sociaux et économiques. Lentement, les rôles se modifient : les hommes commencent à s'impliquer activement dans les soins et l'éducation de leurs enfants tandis que les femmes entrent sur le marché du travail.

Constatant qu'après le divorce, les femmes obtiennent la garde des enfants dans presque tous les cas, plusieurs hommes se sentent bafoués dans leurs droits. Ils ne veulent plus être réduits à leur rôle de pourvoyeurs ou de visiteurs de fins de semaine. Ils soutiennent que les deux parents devraient avoir la même possibilité d'obtenir la garde de leurs enfants au moment de la séparation, puisqu'ils sont tous deux en mesure de répondre à leurs besoins. Ils accusent le système judiciaire de fonder ses jugements sur des valeurs sexistes et conservatrices, ou sur des croyances sans fondement, telle la doctrine de l'âge tendre (Roth, 1984).

Les dernières décennies voient apparaître plusieurs associations d'hommes dont le but est de faire valoir les droits des pères divorcés à prendre de plus grandes responsabilités dans l'éducation de leurs enfants ${ }^{3}$. Ces revendications masculines joueront un rôle majeur dans le changement de la loi californienne de 1979, premier État américain à statuer que la garde conjointe est la forme de garde qui répond le mieux aux intérêts de l'enfant. 
En résumé, la garde des enfants au moment de la séparation repose sur une philosophie et sur des valeurs acceptées par une société à un moment donné de son évolution. Comme l'une des plus nobles fonctions de la loi est de traduire ces valeurs en établissant des modèles de comportements à suivre par les citoyens, nous verrons comment les lois (et particulièrement les lois canadiennes) se sont adaptées à ces changements sociaux en favorisant à divers degrés l'implication des deux parents dans l'éducation des enfants.

Du changement de mentalité au changement de la loi. Comme nous le verrons, d'un pays à l'autre et à travers le temps, les lois concernant la garde des enfants se sont progressivement modifiées.

C'est apparemment en 1964, en Angleterre, que l'on adressa pour la première fois aux tribunaux une demande qui consistait à attribuer la garde juridique aux deux parents, avec garde physique à l'un d'eux et droits de visite et de sortie à l'autre. Le juge refusa cette demande. Mais, quelques années plus tard, on vit apparaître quelques-unes de ces gardes conjointes (L'Heureux-Dubé, 1979).

Aux États-Unis, en 1979, la Californie est le premier État à légiférer par rapport à la garde conjointe. La loi, amendée en 1983, exprime clairement que la politique de l'État est d'assurer aux enfants mineurs des contacts fréquents et continus avec leurs deux parents, après la séparation ou le divorce de ceux-ci (California Civil Code, s. 4600, a).

Conséquemment, quand les deux parents s'entendent, la présomption en faveur de la garde conjointe est établie (California Civil Code, s. 4600, 5, a). On considère que c'est la forme de garde qui répond le mieux aux intérêts des enfants mineurs. Quand les parents ne s'entendent pas, il est établi que la garde conjointe est une option tout aussi valable que la garde unique (California Civil Code, $s$. $4600, b, 1)$, et si le juge ne recommande pas la garde conjointe, il doit en mentionner les raisons dans son jugement. De plus, en accordant la garde unique à l'un des parents, il doit considérer lequel des deux parents sera le plus en mesure de permettre à l'enfant des contacts fréquents et continus avec l'autre parent.

Cette législation avant-gardiste a un effet d'entraînement sur les autres États américains. Certains, comme la Floride, vont même jusqu'à statuer que la Cour doit ordonner la garde conjointe, à moins qu'elle puisse prouver que cette forme de garde serait au détriment de l'enfant. Aujourd'hui, environ trente-deux États américains ont adopté une législation reconnaissant la légalité de la garde conjointe; les lois varient cependant quant à la volonté d'imposer cette garde à des parents qui ne s'entendent pas (Ryan, 1986). 
Au Canada, quelques notions sur l'évolution des lois en matière de divorce nous aideront à mieux saisir les changements dans les lois et les mentalités relativement à la garde des enfants.

Avant 1837, le Code civil canadien français n'admettait pas la dissolution du mariage, et les femmes mariées n'avaient pas de statut juridique (Statistique Canada, 1983). Au moment de la Confédération, on attribua au Parlement du Dominion la compétence exclusive en matière de divorce. À cet égard, le gouvernement fédéral devait établir des tribunaux dans les provinces, mais il s'abstint de le faire, laissant à celles-ci le choix de légiférer à leur gré. Au Québec, où la religion catholique imposait son dogme de l'indissolubilité du mariage, il fallut attendre jusqu'en 1968 avant de voir apparaître une loi provinciale en matière de divorce. Aussi, les résidents du Québec désireux "malgré tout » d'obtenir un divorce devaient s'adresser au Parlement fédéral pour obtenir un bill privé.

L'organisation de base des relations entre les époux a été profondément modifiée depuis quelques années. Par les réformes de 1964, 1969 et 1981, on est passé d'un régime de complète subordination de la femme à un régime d'égalité entre époux (D.-Castelli, 1987). Ces modifications ont entraîné des changements par rapport à la garde des enfants.

Ainsi, jusqu'à la promulgation de la nouvelle loi sur le divorce en 1985, les époux étaient obligés de s'accuser l'un l'autre pour obtenir le divorce. Ce système accusatoire se reflétait au niveau de la garde des enfants. Lors des ruptures de couples, le juge devait décider de la garde sur la base de la preuve partisane des parents (C.S.F. 1985). Le service d'expertise psychosociale, qui cherchait à déterminer le meilleur intérêt de l'enfant, n'intervenait qu'en cas de conflit et ne faisait pas partie d'un processus de conciliation préventif. Le service de conciliation à la famille en vue d'aider les conjoints à parvenir à des décisions concernant les mesures accessoires du divorce, dont la garde des enfants, ne sera mis sur pied qu'en 1981, à Montréal.

Mais qu'en est-il du statut légal de la garde conjointe ? Quelques clarifications s'imposent. D'abord, selon le Code civil, la garde de l'enfant est un droit (ou un attribut essentiel) qui découle logiquement de la notion d'autorité parentale. Cette autorité vient de la nécessité pour les parents d'assurer à l'enfant les soins, l'éducation et l'intégration sociale.

Jusqu'en 1977, l'autorité sur les enfants appartenait au père et portait alors le nom de "puissance paternelle ». Souvenons-nous que le mari était reconnu comme le chef de famille et que c'est lui qui détenait l'autorité sur les enfants, celle de la mère ne devenant effective qu'au décès du père (D.-Castelli 1987, p. 178). 
Depuis 1977, l'autorité parentale appartient aux deux parents qui ont donc un droit égal de garde, de surveillance et d'éducation (la seule limite à cette autorité étant la Loi de la protection de la jeunesse). Au moment du divorce ou de la séparation, tous deux restent, et d'une manière égale, titulaires de l'autorité parentale. Tous deux ont donc, et d'une manière similaire, les droits de garde de leurs enfants (D.-Castelli, 1987, p. 186).

Cependant, après le divorce, si la garde physique est confiée à I'un des parents, l'autre ne peut exercer son autorité parentale que par le biais de ses droits de surveillance. Et ceux-ci peuvent être grandement limités par le peu de contacts avec l'enfant que permettent les visites de fins de semaine.

Au plan légal, le terme " garde conjointe " pouvait donc porter à confusion puisque, comme le soulignait Claire L'Heureux-Dubé (1979), la notion que ce terme recouvre est, selon notre Code civil, celle d'autorité parentale.

Devant les nouvelles coutumes développées par les ex-conjoints concernant la garde de leurs enfants, les tribunaux ont eu à s'adapter et à préciser le sens de la garde conjointe. Pour eux, cette notion comporte l'attribution de la garde juridique et physique aux deux parents (diverses modalités de la garde physique pouvant être adaptées aux besoins de chacun). Ils entendent donc par garde conjointe le maintien du partage des responsabilités parentales. Cela implique que toutes les décisions importantes concernant I'enfant (sauf celles liées à la vie quotidienne) seront prises par les deux parents. De plus, par la nouvelle loi sur le divorce (1985), les tribunaux canadiens ont aussi défini les conditions nécessaires à l'attribution de cette forme de garde.

"Lorsqu'il prend une décision en matière de garde ou d'accès, le tribunal tient compte uniquement de l'intérêt de l'enfant. L'intérêt de l'enfant comprend d'ordinaire le maintien du contact avec les deux parents. (...) La conduite passée de la personne qui demande la garde ou le droit d'accès n'entre pas en ligne de compte, à moins que cette conduite ne soit liée à la capacité de cette personne de servir de père ou de mère. Toutefois, le tribunal tient compte de la mesure dans laquelle chacun des époux est disposé à favoriser à l'autre le contact avec l'enfant. Le conjoint qui n'est pas disposé à faciliter ce contact pourrait perdre la garde de l'enfant " (Ministère de la Justice, 1986, p. 20).

Comment les tribunaux déterminent-ils « le meilleur intérêt de l'enfant "? Selon madame D.-Castelli :

"Le tribunal écoutera la preuve apportée par chacun des parents. Ceux-ci pourront essayer de déterminer l'intérêt de l'enfant par tous les moyens. Chaque parent réclamant la garde de son enfant 
va essayer de prouver qu'il peut répondre aux besoins matériels et affectifs de l'enfant d'une manière plus valable que l'autre. II essaiera donc de prouver les faits visant à démontrer ses propres qualités et les lacunes de l'autre et de faire établir un lien entre ces faits ou ces attitudes, et un effet positif ou négatif sur l'enfant. (...) Les parents peuvent toutefois chercher non seulement à obtenir à tout prix la garde de leur enfant, mais aussi la solution la meilleure pour ce dernier. Ils pourront alors recourir à l'expertise psychosociale » (1987, p. 187).

Force est de constater que le système accusatoire est maintenu ! Malgré la volonté explicite des tribunaux de contribuer à maintenir pour l'enfant la relation avec ses deux parents, la législation canadienne n'accorde pas une priorité favorable à la garde conjointe. Au contraire, "Comme la garde conjointe exige que les personnes en cause s'engagent à collaborer pour prendre les grandes décisions concernant l'éducation de l'enfant, son lieu de résidence, les soins médicaux et ainsi de suite et soient en mesure de le faire » (Ministère de la Justice, 1986, p. 21). La garde conjointe $n^{\prime}$ est pas reconnue comme étant une option valable quand il y a litige entre les conjoints.

En résumé, la nouvelle loi canadienne sur le divorce de 1985 permet d'accorder la garde conjointe si les deux parents le veulent, mais maintient le système adverse en cas de conflit. Aucun droit de parole officiel n'est accordé à l'enfant et le juge reste libre de sa décision puisqu'il n'est tenu ni par la décision du service d'expertise psychosociale, ni par les préférences que l'enfant aurait exprimées (D.-Castelli, 1987, p. 188).

\section{Les enjeux des différentes formes de garde : conséquences sur les enfants, les hommes et les femmes}

Afin de bien saisir quels sont les enjeux psychosociaux impliqués dans les différentes formes de garde des enfants, nous ferons d'abord une revue des recherches scientifiques dans le domaine. Puis, nous examinerons plus spécifiquement les implications économiques.

Conséquences du divorce sur les enfants. Avant de relever les conséquences rattachées aux différents types de garde, il peut être important de s'arrêter aux conséquences du divorce.

Plusieurs personnes pensent que "pour le bien des enfants», il faut absolument éviter le divorce. D'autres vont se demander s'il est préférable pour les enfants de vivre avec des parents en conflit ou de subir une séparation ou un divorce.

De nombreuses études ont montré que lorsque des parents, divorcés ou non, sont en conflit, les enfants présentent un plus grand nombre de problèmes psychologiques. Par exemple, ils peuvent avoir 
une faible estime de soi, plus d'anxiété ou moins de confiance en soi. Selon ces études, il semble que ce $n^{\prime}$ est pas le divorce comme tel qui perturbe les enfants, mais plutôt les conflits entre les parents (Emery, 1982; Porter et O'Leary, 1980). Il est même de plus en plus clair que ni les conflits parentaux ni le divorce ne constituent une condition suffisante ou nécessaire pour qu'il y ait des problèmes psychologiques chez les enfants (Emery et O'Leary, 1984; Hodges, Buchsbaum et Tierney, 1983). Probablement que les conflits maritaux ont des effets négatifs chez les enfants, uniquement lorsque d'autres facteurs de stress sont aussi présents. Ainsi, les difficultés économiques, la qualité de la relation parents-enfants, le fait d'avoir des parents très jeunes et la mobilité géographique semblent être des facteurs importants dans l'explication des problèmes psychologiques des enfants de parents divorcés (Hodges, Tierney et Buchsbaum, 1984). Certains auteurs considèrent même que, lorsque les parents savent réorganiser la situation familiale d'une façon harmonieuse, le divorce peut être un événement positif parce qu'il permet de diminuer la fréquence et l'intensité des conflits familiaux (Felner, Farber et Primavera, 1983).

La recherche ne confirme donc pas l'idée très répandue que les conjoints en conflit doivent nécessairement rester ensemble pour sauvegarder le bien-être de leurs enfants. Lorsque des parents se séparent, il semble que les enfants ne se détériorent pas nécessairement davantage et peuvent même parfois s'en trouver mieux (Emery et O'Leary, 1984; Stuart, 1980).

Après avoir fait une revue de littérature dans le domaine, Gilles Poirier du CSS de Québec mentionne que les enfants qui s'en sortent le mieux sont, ou bien ceux qui peuvent conserver une vraie relation avec leurs deux parents, ou bien ceux dont les parents résolvent leur crise et s'en sortent de façon «positive ». L'enfant est parfois davantage influencé par la façon dont les parents vivent leur divorce que par la séparation elle-même (Poirier, 1981; Fortin, 1985).

Quelles sont donc maintenant les conclusions des recherches qui comparent la garde unique à la garde conjointe ?

\section{Les conséquences reliées aux différents types de garde des enfants}

En 1975, Wallerstein et Kelly effectuent une étude sur les conséquences des différents types de garde auprès de 131 enfants. Ils soulignent l'importance pour l'enfant d'âge préscolaire de maintenir des contacts continus avec les deux parents. Ils révèlent que les enfants réellement satisfaits étaient ceux qui avaient la possibilité de voir leur père en tout temps, en se rendant, par exemple, à son logis, 
s'ils le désiraient. De plus, ils ont trouvé un lien significatif entre la dépression chez l'enfant et le contact appauvri ou perturbé avec le parent non-gardien.

Grief (1979) va dans le même sens en recommandant de maximiser les contacts parents-enfants. L'une des façons de le faire, selon elle, serait d'encourager les parents à prendre soin de leurs enfants. Ainsi, les pères qui ont une responsabilité conjointe s'impliqueraient davantage dans tous les aspects développementaux de leurs enfants, ce qui contribuerait à maintenir un lien significatif primordial tant pour eux que pour les enfants.

Dans une importante recherche sur la garde partagée, Steinman (1981) étudie un échantillon de 24 familles ayant la garde partagée de 32 enfants âgés entre quatre ans et demi et quinze ans. Elle conclut que les enfants, à l'exception des très jeunes, n'étaient pas confus par rapport à leur calendrier de garde partagée et s'étaient bien adaptés à ce mode de vie. Par ailleurs, ces enfants exprimaient des sentiments " hyper-loyaux » vis-à-vis leurs parents : ils étaient préoccupés par le fait que les deux parents reçoivent autant de temps et d'affection l'un que l'autre.

Malgré plusieurs avantages reliés à la garde partagée, Steinman soulève les difficultés que peuvent éprouver les enfants qui doivent vivre dans deux maisons. Elle rapporte qu'un tiers des enfants, spécialement les filles âgées de 4-5 ans et les garçons de 7-9 ans, avaient de la difficulté à faire face aux exigences qu'impliquent les constants déménagements. De plus, elle souligne que les adolescentes rapportaient des difficultés à maintenir des relations avec leurs ami(e)s. Malgré tout, la plupart des enfants appréciaient la garde conjointe parce que cela leur donnait accès aux deux parents et leur permettait de se sentir aimés par les deux.

Dans une récente recherche, Luepnitz (1986) compare les effets de différentes formes de garde sur 91 enfants de 44 familles (garde conjointe, garde confiée à la mère et garde confiée au père) pendant une période moyenne de trois ans et demi. Elle fait ressortir que la plupart des 91 enfants n'étaient pas inadaptés (seulement huit éprouvaient des problèmes). II est intéressant de constater que leur ajustement était indépendant du type de garde alors qu'il était en lien direct avec le climat de conflit entre les parents. Cependant, les enfants en garde conjointe maintenaient un lien filial avec les deux parents alors que les enfants en garde exclusive n'entretenaient qu'un lien avunculaire avec le parent qui n'avait pas la garde. D'après Luepnitz, la garde conjointe "à son meilleur " est supérieure à la garde par un seul parent "à son meilleur ".

Afin de vérifier les effets à long terme du divorce sur les relations parents-enfants, Fine et Worley (1986) comparent les relations 
parentales entre deux groupes d'étudiants. Un premier groupe de 149 étudiants dont les parents ont divorcé 18 ans plus tôt est comparé à un deuxième groupe de 153 étudiants provenant de familles dites " intactes" (sans divorce). Les résultats confirment que les sujets de familles divorcées perçoivent de façon moins positive la qualité de leur relation avec leur père que les sujets des familles intactes. Suite au divorce, ces sujets avaient été confiés à la garde de leur mère. Aussi, les conflits entre les ex-conjoints et le manque d'opportunité de contacts quotidiens entre les pères et leurs enfants peuvent avoir contribué à diminuer l'implication des pères auprès de leurs enfants. Selon cette recherche, les facteurs qui prédisent le mieux la qualité de la relation parent-enfant après le divorce sont la qualité de la relation avant le divorce, l'ajustement de l'enfant et la qualité de la relation entre les ex-conjoints.

En somme, d'après bon nombre de recherches, il appert que la garde conjointe où les parents maintiennent un climat de collaboration est favorable aux enfants car elle leur permet de maintenir une relation signifiante avec leurs deux parents. Quant aux conséquences économiques sur les enfants, nous en examinerons les implications par le biais des conséquences sur les femmes.

\section{Les conséquences de la garde conjointe sur les parents}

Dans la majorité des recherches, les parents rapportent une plus grande satisfaction face à la garde physique conjointe des enfants que face à la garde par un seul parent (Ahrons, 1979; Luepnitz, 1983; Rosenthal et Keshet, 1981). Certains auteurs (Rothberg, 1983) soulignent que des parents demeurent ambivalents devant les difficultés que peuvent éprouver les enfants dans leurs déménagements continus ou devant le fait de devoir maintenir une relation avec leur ex-conjoint. Toutefois, ces données ne sont pas constantes dans la littérature. Par exemple, Luepnitz (1983) rapporte que les parents ayant une garde partagée éprouvaient moins de conflits avec leur ex-conjoint que les parents ayant une garde unique. Selon elle, cela ne signifie pas que les conjoints ayant une garde partagée $n^{\prime}$ avaient pas de conflits mais qu'ils étaient capables $d^{\prime} y$ faire face d'une manière plus civilisée que les autres ayant une garde unique. On pourrait alors se demander si la garde partagée n'est pas déjà un indice du climat plus positif qui existe entre ex-conjoints.

Le taux de "relitigation", soit le fait de retourner à la Cour pour demander des modifications à l'entente établie, est beaucoup plus bas chez les conjoints ayant une garde conjointe que ceux ayant une garde exclusive. Ayant suivi 414 cas de garde en Californie, Ilfeld et ses collaborateurs (1982) parlent d'un taux $50 \%$ plus bas et Luepnitz 
(1986) mentionne qu'aucun couple de sa recherche ayant une garde partagée n'est retourné à la Cour tandis que $56 \%$ des parents en garde exclusive y sont retournés au moins une fois pour des questions financières ou de droit de visite. On peut se demander si la garde conjointe favorise le maintien de relations interpersonnelles suffisamment satisfaisantes pour que les conjoints n'aient pas à retourner devant la Cour... ou encore si ceux qui optent pour cette forme de garde ne sont pas tout simplement en mesure de régler leurs problèmes "à l'amiable "... Peut-être aussi que le taux de "relitigation " n'est pas un indice valable du taux de conflits entre les parents (Richards et Goldenberg, 1985).

Les conséquences sur les hommes. Probablement dû à la relative nouveauté du phénomène homme-père-chef de famille monoparentale, bon nombre de recherches se sont penchées sur les conséquences des différents types de garde sur les hommes. Par rapport à la garde conjointe, les pères rapportent un accroissement de leur sentiment d'estime d'eux-mêmes et de leur capacité de s'occuper adéquatement de leurs enfants (Grief, 1979; Luepnitz, 1983; Richards et Goldenberg, 1985; Rosenthal et Keshet, 1981; Steinman, 1981). Plusieurs changent leurs valeurs en accordant moins d'importance à leur travail qu'à la relation avec les enfants et passent souvent plus de temps avec eux qu'avant le divorce.

Ces données contrastent avec celles recueillies auprès des pères qui n'ont pas la garde de leurs enfants. Contrairement au mythe populaire voulant que les pères deviennent libres en divorçant, il s'avère que dans les faits, la majorité des hommes subissent un stress assez marqué et suffisamment sérieux pour générer des problèmes physiques. À la suite de la séparation, $33 \%$ des hommes étudiés par Greif (1979) ont développé des symptômes physiques tels qu'une perte de poids importante et soudaine allant de 8 à 50 livres, des problèmes visuels et dentaires diagnostiqués comme consécutifs à la nervosité, une pression sanguine élevée, des rhumatismes, de l'arthrite ainsi que des troubles psychosomatiques. On rapporte également une augmentation de la consommation d'alcool ainsi que des changements dans la performance sexuelle.

Dans un rapport sur différentes recherches effectuées en vue d'améliorer la situation des hommes séparés ou divorcés et celle de leurs enfants (L'Espérance, Gentile, Lee, 1984), I'Association des hommes séparés ou divorcés de Montréal va dans le même sens. Les auteurs soulignent les effets néfastes (tant sur les pères que sur leurs enfants) qu'engendre la garde unique confiée à la mère. Dans ces situations, le père n'est présent que les fins de semaine et, très souvent, à long terme, la qualité de la relation avec ses enfants se 
détériore. Aussi, cette association suggère-t-elle de favoriser plus ouvertement la garde conjointe.

Si nous examinons les implications économiques reliées au divorce, la situation semble cette fois tout à l'avantage des hommes. En effet, dans la majorité des cas de divorce, les femmes obtiennent la garde des enfants, mais leur niveau de vie ainsi que celui de leurs enfants périclite alors que celui des hommes s'améliore. Selon un récent document du Conseil consultatif canadien sur la situation de la femme (1987), les hommes ont presque le double de revenu net après la séparation comparativement à celui qu'ils avaient avant. Cela est dû à différents facteurs : la place des hommes évidemment plus avantageuse sur le marché du travail; le manque de barèmes objectifs pour fixer la pension alimentaire; l'octroi de pensions alimentaires souvent infimes et déductibles d'impôts, réajustables selon l'augmentation des besoins de l'homme; le refus pour plusieurs hommes de payer ces pensions et, son corollaire, la faiblesse du système de perception des pensions.

Parmi les recherches sur les conséquences du divorce et des différents types de garde, la plupart étudient uniquement les conséquences psychologiques; très peu tiennent compte des implications économiques. Toutefois, parmi celles que nous avons pu identifier, les résultats vont dans le même sens. Par exemple, d'après une recherche effectuée auprès de 228 personnes divorcées aux ÉtatsUnis, Weitzman (1981) indique que les femmes connaissent une baisse de leur niveau de vie de l'ordre de $73 \%$ après le divorce, tandis que leurs maris voient le leur augmenter de $42 \%$.

Par ailleurs, portant une attention particulière aux conditions économiques des parents qui font l'expérience de la garde conjointe, certains professeurs des facultés de service social et de sociologie de I'Université de Toronto (Irving et Benjamin 1986, p. 79-103) observent que les parents qui partagent la garde ne sont pas nécessairement riches (le tiers de leur échantillon ont un revenu annuel inférieur à $20000 \$$ et $26 \%$ d'entre eux sont de niveau secondaire ou moins). Selon l'interprétation qu'ils font de leurs données, il serait injuste de soutenir que la garde conjointe ne peut être expérimentée que par des riches, puisque $86 \%$ de leurs répondants ne croient pas que les coûts reliés à ce type de garde ou que les problèmes financiers soient une source majeure de conflits entre eux. Pourtant, en corollaire, nous devons souligner que ces données indiquent aussi que $67 \%$ de leurs répondants gagnaient entre $21000 \$$ et $60000 \$$ annuellement et que $74 \%$ d'entre eux avaient un niveau d'éducation post-secondaire ou universitaire. Il faudrait sans doute poursuivre ces recherches et voir si la garde conjointe permet une plus juste répartition des richesses entre les hommes et les femmes ou si elle n'est pas le 
nouvel alibi pour permettre aux hommes de diminuer la pension alimentaire.

Les conséquences sur les femmes. L'impact sur les femmes des différents types de garde a reçu moins d'attention dans la littérature. Cependant, à travers les études comparatives, les aspects les plus avantageux relevés par les femmes ont trait surtout au partage des responsabilités parentales incluant la prise de décision, les soins réguliers à donner aux enfants et la question monétaire (Nehls, 1981; Luepnitz, 1983, 1986). Par exemple, Luepnitz (1986) rapporte que seulement la moitié des mères ayant la garde exclusive ont reçu un soutien financier adéquat de la part de leur conjoint tandis qu'aucune, ayant une garde conjointe, n'est retournée devant la Cour pour faire augmenter la pension.

Mais, qu'en est-il de la situation des Québécoises ? Une enquête du Ministère de la Justice effectuée en 1986 rapporte que moins de $7 \%$ des couples désunis partagent la garde des enfants sous une forme ou sous une autre (Tremblay, 1987, p. 20-22). Près de la moitié des mères qui ont la garde des enfants (et elles l'ont dans $80 \%$ des cas) demeurent au foyer, sans aucun revenu d'emploi. Elles doivent donc vivre d'une pension alimentaire qui n'est pourtant octroyée par une ordonnance légale que pour $42 \%$ des couples avec enfants. De plus, ces pensions sont très peu élevées (la moitié d'entre elles ne dépassent pas 260 \$ par mois et le montant mensuel moyen n'est que de 368 \$) et, dans les deux tiers des cas, elles ne sont pas indexées au coût de la vie.

Les données de l'enquête révèlent par ailleurs que $63,5 \%$ des femmes touchent leur pension sans difficulté, 13,8\% ne reçoivent qu'une partie de leur dû, et $22,7 \%$ ne le voient jamais ! Les hommes qui ne paient pas ne sont pas seulement les chômeurs et les assistés sociaux, car 83 \% des mauvais payeurs ont un revenu d'emploi.

Le Québec possède un service de perception alimentaire qui pourrait pallier à cette situation, mais seulement $20 \%$ des femmes qui pourraient y avoir recours le font. Les autres invoquent des raisons telles que la durée et la complexité des procédures, le manque présumé d'efficacité et la peur des représailles de leur ex-conjoint.

Un grand nombre de femmes vivent sous le seuil de la pauvreté, spécialement celles qui vivent avec de jeunes enfants, après un divorce. La collaboration financière soutenue du conjoint est extrêmement importante pour les femmes, et constituerait pour elles un des avantages majeurs liés à la garde conjointe.

Cependant, il ne faudrait pas affirmer trop vite que la garde conjointe est «la » solution à tous les problèmes. Est-ce juste que les femmes qui n'ont pas les mêmes revenus que les hommes partagent à part égale les frais de garde? Certaines avocates féministes (amé- 
ricaines) soutiennent que la garde conjointe n'est peut-être pas avantageuse pour les femmes dont le revenu est peu élevé et dont les possibilités d'avancement sont réduites. Dans ces cas, devenir mère à temps partiel peut être perçu comme une perte. Dans le même sens, deux juristes américains (Mnookin et Kornhausser, 1979) soutiennent que les femmes tiennent ordinairement plus que les hommes à garder leurs enfants et que cela donne un plus grand pouvoir de négociation aux hommes. Il en résulte qu'il n'y a pas vraiment plus d'Américains qui s'occupent de leurs enfants qu'autrefois, qu'ils paient en moyenne moins de pensions alimentaires et versent de moins en moins de pensions aux femmes (Legault et Pineault, 1985, p. 31).

La même tendance se dessine au Canada. Ainsi, au cours des années 1970 et au début des années 1980, afin de fixer les pensions alimentaires pour les femmes, les juges ont eu tendance à invoquer le principe de la " rupture nette » en vertu duquel les ex-conjointes qui n'étaient pas financièrement autonomes se voyaient accorder de une à trois années pour le devenir, peu importe depuis combien de temps elles avaient quitté le marché du travail et à quel point leurs compétences étaient désuètes. Cette approche impitoyable a été renversée par la Cour suprême en 1983 en décrétant que la pension devait être versée jusqu'à ce que l'ex-épouse ait effectivement acquis les moyens de subvenir à ses besoins (CCCSF, 1987, p. 31). II demeure que les critères d'évaluation dans ce domaine ne sont pas faciles à préciser. Aussi, il est évident qu'une véritable justice sociale pour les femmes dépendra de politiques sociales autres que le maintien à long terme de la pension alimentaire.

Enfin, nous ne pouvons pas parler des conséquences des différents types de garde sur les femmes sans parler de la violence familiale dont près de $95 \%$ des victimes sont des femmes (Comité de la consultation sur la politique familiale, 1987, p. 127). Il est important de rappeler que la violence conjugale n'est pas rare. Aux États-Unis, certains experts affirment que $50 \%$ des femmes seront battues à un moment donné de leur vie par un homme "qui les aime " (Walker, 1979). Même les estimations les plus conservatrices, basées sur des échantillons représentatifs de la nation américaine, estiment qu'environ $30 \%$ des femmes mariées ont vécu une forme quelconque de violence physique de la part de leur conjoint. Au Canada, on estime qu'une femme sur 10 est battue dans une union légale ou de fait (McLeod et Cadieux, 1980) et on peut supposer que le même rapport existe pour le Québec (Pâquet-Deehy et al., 1985). Au moment du divorce, ces femmes (et leurs enfants) ont besoin de protection. Pour certaines, il est essentiel que leur sécurité soit assurée en coupant tout contact avec leur ex-conjoint abusif et, dans ces cas, la garde exclusive s'impose (Luepnitz, 1983). Mais encore là, cette forme de 
garde ne peut pas être la seule mesure de sécurité : plusieurs recommandations relatives aux politiques sociales dans ce domaine doivent se concrétiser dans un avenir prochain.

En résumé, dans un grand nombre de cas, la garde conjointe semble offrir plusieurs avantages aux enfants et aux parents, en particulier une plus grande implication émotive et financière du père de même que le partage des responsabilités et des soins à apporter aux enfants. Par contre, les femmes semblent encore assumer une large part des responsabilités familiales et les implications économiques du divorce jouent à leur désavantage.

\section{Conclusion}

L'histoire du concept de garde montre que l'organisation sociale autour de l'éducation et des soins à apporter aux enfants repose sur des valeurs et des conditions de vie particulières à une société, à un moment donné de son histoire. La revue de la littérature scientifique sur les conséquences des différents types de garde souligne que ce n'est pas tant la structure ou le mode d'organisation de la garde qui est important mais le climat de collaboration entre les parents et les bonnes relations parents-enfants.

À cet égard, le grand nombre de divorces ne révèle-t-il pas que les hommes et les femmes d'aujourd'hui sont à la recherche de nouvelles formes de relations interpersonnelles plus satisfaisantes et libératrices tant pour eux que pour leurs enfants ? Le modèle de la famille traditionnelle éclate de toutes parts. On voit apparaître de nouvelles formes d'unions, sans reconnaissance légale, qui donnent naissance à des enfants. Ceux-ci vivront bientôt sous un autre toit, avec d'autres personnes que le père ou la mère biologique, avec d'autres enfants qui ne sont pas leurs frères ou leurs sœurs, rendant visite à de plus en plus de " grands-parents » ou d'ami(e)s qui ne sont plus des oncles et des tantes. Notre langage n'a pas encore trouvé de nom pour désigner ces nouvelles «familles "... N'est-ce pas un signe évident des profondes mutations qui marquent la fin d'un siècle ?

Cependant, force est de constater que le poids du changement pèse lourd sur les épaules des femmes. Ne sont-elles pas les plus nombreuses parmi les plus démunis? Comment pourront-elles concilier leurs responsabilités parentales (assumées très souvent seules) et leur participation de plus en plus grande sur le marché du travail ?

Penser à la société de demain provoque encore un certain nombre de questions auxquelles nous n'avons pas fini de réfléchir... Quelle sorte de projet de société désirons-nous par rapport à l'éducation et aux soins à apporter aux enfants ? Que signifie pour 
nous "le meilleur intérêt de l'enfant " ? Comment définirons-nous les rôles des hommes et des femmes ? Quel rôle l'État devra-t-il jouer, et comment pourra-t-il le faire ?

Le désir d'une plus grande justice sociale dans le partage des responsabilités parentales n'est sans doute pas le seul apanage des femmes. Cependant, le retard à adopter des mesures efficaces se fait actuellement au détriment de plusieurs citoyens, et en particulier des femmes et des enfants. Pour que les choses changent plus rapidement, les femmes devront prendre plus de pouvoir, continuer à modifier leur environnement et contribuer à ce que des lois et des mesures sociales soient votées et mises en place, tant à leur avantage qu'à celui des enfants dont elles sont en grande partie responsables.

\section{Notes}

${ }^{1}$ Le Québec est la province canadienne où la plus forte diminution des mariages a été enregistrée; entre 1972 et 1986, elle a été de 42,7\%. "Pas populaire le mariage!", La Presse, 27 octobre 1987 (citant Statistique Canada, 1987).

${ }^{2}$ Certains des facteurs mentionnés se retrouvent dans le Rapport du comité de la consultation sur la politique familiale, deuxième partie, Le soutien collectif réclamé pour les familles québécoises, 1987, p. 18.

${ }^{3}$ Raymond Denis, du collectif Hom-Info, faisait valoir qu'un père qui désire s'intéresser à l'éducation de ses enfants voit son rôle réduit par les tribunaux à celui de pourvoyeur et de père de fins de semaine. (La Presse, 4 novembre 1984).

\section{Bibliographie}

Ahrons, C. (1979). The coparental divorce : Preliminary research findings and policy implications. Joint Custody: A handbook for judges, lawyers, and counsellors. Ft. Lauderdale : The Association of Family Conciliation Courts, Nova University Law Center.

CALIFORNIA CIVIL CODE, s. 4600 (a).

Conseil consultatif canadien sur la situation de la femme (1987). Participation et Intégration. Les femmes, le travail et l'argent, Ottawa.

Conseil du statut de la femme (1985). Oui, Mais... Le bilan des activités gouvernementales en matière de condition féminine est-il positif? Bibliothèque nationale du Québec, 52.

COMITÉ DE LA CONSULTATION SUR LA POLITIQUE Familiale (1987). Le soutien collectif réclamé pour les familles québécoises et Le soutien collectif recommandé pour les parents québécois. Québec : gouvernement du Québec.

D.-CAStelLI, M. (1987). Précis du droit de la famille, Bibliothèque juridique, Les Presses de l'Université Laval.

Derdern, A. (1978). "Child Custody Contests in Historical Perspective", American Journal of Psychiatry, 12, 133. 
ElKIN, M. (1987). "Joint Custody : Affirming that Parents and Families are Forever ", Social Work, 32, 1, 18-24.

EMERY, R.E. (1982). Interparental conflict and the children of discord and divorce. Psychological Bulletin, 310-330.

EMERY, R.E., O'LEARY, K.D. (1984). Marital discord and child behavior problems in a nonclinic sample. Journal of Abnormal Child Psychology, 12, 411-420.

Felner, R.D., Farber, S.S., Primavera, J. (1983). Transitions and stressful life events : A model for primary prevention. In R.D. FELNER, L.A. JASON, J.N. MORItSUGu et S.S. FARBER (Eds), Preventive psychology: Theory, research, and practice. New York: Pergamon.

FINE, M.A., WORLEY, S.M. (1986). The Effects of Divorce on Parent-Child Relationships. Journal of Social Behavior and Personality, 1, 3, 451-463.

Fortin, A. (1987). Histoire de familles et de réseaux : la sociabilité au Québec $d^{\prime}$ hier à demain, Les Éditions Saint-Martin, Montréal.

FORTIN, D. (1985). L'entente de garde conjointe suite aux interventions du Service de médiation à la famille de Montréal. Mémoire de maîtrise es sciences, Service Social, Université de Montréal.

Gauthier, P., Boyer-Cayouette, D., Dumais-Charron, L., Fortin, C., Gosselin, L. et HOTTE, J.P. (1982). Mères et enfants de familles monoparentales. École de psycho-Éducation, Université de Montréal.

Guıck, P.C. (1979). "Children of Divorce. Parents in Demographic Perspective ", Journal of Social Issues, 35, 170-182.

GRIEF, J. Fathers, Children and Joint Custody. (1979). American Journal of Orthopsychiatry, 49, 2.

Hodges, W.F., BuChsbaum, H.K., Tierney, C.W. (1984). Parent-child relationships and adjustment in preschool children in divorced and intact families. Journal of Marriage and the Family, 46, 611-617.

HOdGes, W.F. TierneY, C.W. BUChsBaum, H.K. (1983). The cumulative effect of stress on preschool children of divorced and intact families. Journal of Divorce, 7, 43-58.

Ilfeld, F., Ilfeld, H., Alexander, J. (1982). Does joint custody work ? A first look at outcome data of relitigation. American Journal of Psychiatry, 139, 62-66.

IRving, H., Benjamin, M. (1986). Shared Parenting in Canada: Questions, Answers and Implications, Family Law Quartely, 1.

Keshet, H.F. (1978). Rosenthal, K.M. "Fathering after Marital Separation ", Social Work, 23, January, 11-18.

Kurdek, L.A. (1983). Children and Divorce, Ed. New Directions for Child Development, San Francisco.

LAROCHE, G. (1985). "Garde des enfants. Moi aussi j'ai deux maisons », Revue Justice, mars, 10-15.

LeGault, D., Pineault, D. (1985). La garde partagée, piège ou libération ? La vie en rose, avril, 29-32.

L'ESPÉRANCE, A., Gentile, C., LeE, O. (1984). Rapport sur les recherches pour améliorer la situation des hommes séparés ou divorcés et le bien-être des enfants. Association des hommes séparés ou divorcés de Montréal.

L'HeUREUX-Dubé, C. (1979). La garde conjointe, concept acceptable ou non ? Revue du Barreau, tome 39, $\mathrm{n}^{\circ} 5$, sept-oct.

LuEPNITZ, D. (1983). Child Custody, New York : Lexington Press. 
LUEPNITZ, D.A. (1986). A Comparison of Maternal, Paternal, and Joint Custody : Understanding the Varieties of Post-Divorce Family Life. Journal of Divorce, 9, 3, Spring.

Mcleod, L. et Cadieux, A. (1980). La femme battue au Canada: un cercle vicieux. Conseil consultatif canadien de la situation de la femme, Canada : Approvisionnements et services Canada.

Ministère DE la JUSTICE (1986). La loi sur le divorce. Guide à l'intention des conseillers, Ministère des Approvisionnements et Services, Canada, (1986).

MNOOKIN, R. et Kornhauser, L. (1979). Bargaining in the Shadow of the Law : The Case of Divorce. Yale Law Journal, 88.

NeHts, N. (1981). Joint Custody of Children : A Descriptive Study, Ed. NelsonHall, Chicago.

Pàquet-Deehy, A., Rinfret-Raynor, M., Larouche, G. (1985). Violence familiale : un colloque. Évolution actuelle au plan de la recherche, de l'intervention et de la formation. Revue canadienne de service social, 315-330.

POIRIER, G. (1981). Les enfants et le divorce, une revue de la littérature. Service de la recherche et de la documentation, C.S.S. Québec.

Porter, B., O'LeARY, D. (1980). Marital discord and chilhood behavior problems. Journal of Abnormal Child Psychology, 8, 287-295.

RiCHARDS, C. et GOlDENGERG, I. (1985). Joint Custody : Current Issues and Implications for Treatment. The American Journal of Family Therapy, 13, 4.

ROSENTHAL, K., KeSHet, H. (1981). Fathers without partners : A study of fathers and the family after marital separation. Totowa, N.J.: RowmAN et LITTLEFIELD.

RotberG, B. (1983). Joint Custody : Parental problems and satisfactions. Family Process, 22, 43-52.

Rотн, (1984). The Tender Years Presomption of Joint Custody, Family Law Quartely, 225.

Ryan, J.P. (1986). Joint Custody in Canada : Time for a Second Look. Reports of Family, 49 R.F.L. (2d).

Statistique Canada (1983). Divorce : la loi et la famille au Canada, Ottawa, Ministère des Approvisionnements et services.

StatistiQUe CANADA (1985). La population active, Ottawa : Approvisionnements et Services Canada.

Statistique Canada (1986). L'état de parent seul : Caractéristiques et déterminants. Ottawa : Ministère des Approvisionnements et Services.

Steinman, S. (1981). The experience of children in a joint custody arrangement : A report of a study. American Journal of Orthopsychiatry, 51, 403-414.

StreAN, H. (1983). "Worker-client Relationships ". In Handbook of Clinical Social Work, RosenblatT, A., Waldfoget, D. General Editors. Jossey-Bass Publishers, San Francisco, 266-278.

StUART, R.B. (1980). Helping couples change : A social learning approach to marital therapy. New York : Guildford.

TREMBLAY, O. (1987). "Les maris paient... ", Justice, décembre.

Wallerstein, J., KelLy, J. (1975). The effects of parental divorce : The experience of the preschool child. J. American Acad. Child Psychiatry. 
Wallerstein, J., Kelly, J. (1980). Surviving the Break-up: How Parents and Children cope with Divorce, Ed. Basic Book, New York.

WALKER, L.E. (1977-1978). Battered Women and Learned Helplessness. Victimology : An International Journal, 2.

Weitzman, L.J. (1981). The Economics of Divorce : Social and Economic Consequences of Property, Alimony, and Child Support Awards. UCLA Law Review, 28. 